\title{
Excitation of Bloch Surface Waves in Zero-Admittance Multilayers for High-Sensitivity Sensor Applications
}

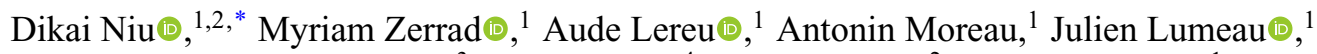 \\ Juan Antonio Zapien $\odot,{ }^{3}$ Ali Passian $\odot,{ }^{4}$ Vincent Aubry, ${ }^{2}$ and Claude Amra ${ }^{1}$ \\ ${ }^{1}$ Aix-Marseille Univ, CNRS, Centrale Marseille, Institut Fresnel, 13397 Marseille, France \\ ${ }^{2}$ PSA Groupe, Direction Scientifique, Centre Technique de Vélizy, route de Gisy, Vélizy-Villacoublay, F-78140, \\ France \\ ${ }^{3}$ Department of Materials Science and Engineering, City University of Hong Kong, Hong Kong, SAR, People's \\ Republic of China \\ ${ }^{4}$ Quantum information Science, Oak Ridge National Laboratory, 1 Bethel Valley Road, Oak Ridge, Tennessee \\ 37830, USA
}

(Received 26 August 2019; revised manuscript received 27 January 2020; accepted 21 April 2020; published 26 May 2020)

\begin{abstract}
The growing need for classical as well as quantum optical sensing places increasingly stringent requirements upon the desired characteristics of the engendered fields. Specifically, achieving superior field enhancement plays a critical role in applications ranging from chem-bio sensing, Raman and infrared spectroscopies to ion trapping and qubit control in emerging quantum-information science. Due to their low optical losses and ability to exhibit resonant field enhancements, all dielectric multilayers are emerging as an optical material system not only useful to classical photonics and sensing but also of potential to be integrated with quantum materials and quantum sensing. The recently introduced concept of zeroadmittance layers [1] within dielectric multilayer materials, enables the creation and control of resonant fields orders of magnitude larger than the exciting field. Here, invoking the zero-admittance concept, we design, fabricate, and characterize an all-dielectric nonabsorbing stack and demonstrate the engendered huge field enhancement. Describing the fields in terms of Bloch surface waves, we connect the surface field to the semiperiodicity in the dielectric domains of the stack. As a specific application of the resonant field, we propose and demonstrate refractive-index sensing for the detection of trace amounts of an analyte. The results include a quantification of the sensitivity of the device with respect to the profile of the exciting field. The experimental results are shown to be in good agreement with theoretical calculations.
\end{abstract}

DOI: 10.1103/PhysRevApplied.13.054064

\section{INTRODUCTION}

Compared to the many effects that arise from lightmatter interactions, collective electronic effects in specific metals via photon or electron excitation of surface plasmons [2-14], generated much interest in the early efforts to achieve confined, enhanced, and/or sensitive fields. Within the optical approaches to plasmon excitation, a frequently employed system that conveniently allows for coupling of photons and plasmons is the planar interface between a thin metal film and a suitable dielectric medium [15-19]. The planar arrangement proved particularly useful since the field bound to the metal surface, while decreasing exponentially with distance to surface, sensitively depends upon the dielectric function of the bounding medium. These properties were found particularly important in several applications, such as the so-called surface plasmon resonance (SPR) sensing. Despite the many

\footnotetext{
*dikai.niu@fresnel.fr
}

intriguing investigations, the losses associated with electronic excitation in metals have been seen as a bottleneck for a number of sensing and imaging applications [20-24]. Interestingly, all-dielectric multilayers have been shown to exhibit surface modes, which feature similar field characteristics as plasmons but without the losses [25-33]. Design and optimization of multilayers made up of a number of deposited dielectric thin films have been recently shown to provide very large resonant field enhancements and sensitivities $[29,34]$. As a result, multidielectric stacks are emerging as an alternative [1] to plasmonic devices for applications, where huge field enhancements in planar multilayers [35-40] are needed. While plasmonic devices provide broadband but moderate enhancements, dielectric stacks provide huge narrowband enhancements. Owing to the mature optical thin-film technologies and associated industries, dielectric stacks also offer a number of key advantages [41-44], including modern deposition systems [45-47] such as dual ion-beam sputtering, and magnetron sputtering. Current capabilities include production 
of amorphous dielectric films with optimal high density, excellent hardness and adhesion, weak optical absorption, and high laser-induced damage threshold.

To advance the optimum design of dielectric stacks, Amra et al. recently introduced the concept of zeroadmittance layers (ZAL) [1]. Employing ZAL, one may analytically design stacks to achieve enhancement for arbitrary wavelengths, polarizations, and incidence angles. The ZAL-based design technique is flexible with respect to the substrate (e.g., a multilayer), and spectral and angular dependencies such that enhancements [1] can be achieved at multiple operational wavelengths and angles. Capitalizing upon the total internal reflection (TIR) mechanism, stacks and related components find numerous applications in the fields of microsources and sensors [20-33]. Therefore, the realization of new resonant stacks designed and optimized through ZAL, is expected to provide excellent candidates for high-sensitivity optical sensors. However, thus far, such stacks have not been fabricated or characterized. It is the aim of this paper to fabricate, measure, and analyze the sensitivity of the ZAL all-dielectric structures. As an application of the optical system, we capitalize on excitation of surface fields via modes that resemble Bloch waves in photonic crystals, to carry out refractive-index sensing.

As with the development of any sensing platform, dissipation and losses of optical energy are important factors that determine the feasibility and suitability of a given sensing mechanism and device. As a major difference with comparable plasmonic sensing strategies, ZAL multilayers are expected to be essentially lossless. The absence of absorption implies that when an incoming field is coupled to a ZAL stack in the TIR regime, the reflectivity $R$ reaches unity, regardless of the illumination conditions (including those of resonance). For that reason, one cannot observe the classical absorption valley, and this is a key difference with plasmonic sensing. However, other remaining criteria can provide a channel for detection, such as light scattering, phase properties, and spatial profile of the reflected beam. For example, scattering from interfacial roughness is proportional to the stationary field and also exhibits enhancement at the resonance. The scattering may thus be collected at the input or the output surface. Similarly, the phase of the fields undergoes a step at the resonance. Here, we explore the reflected beam's profile as this constitutes the simplest measurable criterion.

Our presentation is organized as follows. In Sec. II, within the scope of the present work, we recall the principles of the ZAL design technique. In Sec. III, we use an exact wave-packet calculation to analyze the angular or spatial shape of a Gaussian beam reflected by a ZAL multilayer. Sections IV and V are devoted to the fabrication and metrology of the coatings, as well as to validating all predictions. In Sec. VI we quantify the sensitivity of the technique before concluding in Sec. VII.

\section{ZERO-ADMITTANCE LAYERS}

All-dielectric multilayers are lossless stratified media, which are known to allow tailoring of light properties (spectral, spatial, and temporal shape) with numerous degrees of freedom [41-44]. Mostly used in free space, these materials provide a variety of passive optical functions including antireflection coatings, beam splitters, dichroic and polarizers, narrowband filters, broadband and chirped mirrors [41-44]. Prior to describing the ZAL principles, we consider the following preliminaries.

\section{A. Propagating and evanescent fields at surfaces of periodic material domains}

Prior to presenting our analysis of the fabricated dielectric system, it is prudent to address the terminology employed to describe the interaction of light with the multilayer system. The description of materials with finite or short-range periodicity in its atomic or molecular arrangements, or in its specific arrangement of dielectric domains can benefit from the formulation and treatment of crystals in condensed matter. An example is the photonic crystal, which is made up of mixed high- and low-index dielectric domains. Drawing analogies from condensed-matter crystals, one poses the question of how to control the properties of the material to achieve control over its photonic response, that is, "light-dielectric stack" interaction.

Dielectric stacks or multilayers constitute a class of materials that may be characterized as a one-dimensional (1D) quasiperiodic system. In the present case, the planar material domains are of infinite extent compared to the wavelength of the interacting field, and thus a onedimensional formulation is sufficient. Considering the short-range periodicity in one dimension (in the $z$ direction perpendicular to the thin-film planes), the analogy still proves to be illuminating. Dispersion, band gaps, and Bloch waves are helpful in the description of any wave phenomena in a periodic or nearly periodic potential. A ZAL optical system bears close resemblance to a photonic crystal, which designates a low-loss dielectric medium with periodicity in its material constituency. A photon's propagation through the photonic crystal obeys specific dispersion and band gap. Thus, under proper design conditions, light of specific frequency and direction entering the multilayer system may not be allowed to propagate.

Additionally, many material domains, such as stacks, exhibit surface modes. By a surface wave [48], it is implied that the wave is bound to the dielectric-dielectric interface (including dielectric-vacuum or air) and decays exponentially away from the boundary. For a dielectric-air interface, at the input side (dielectric), an index-guided mode at a critical angle can describe the TIR, while at the air output boundary, the index-guided mode can create an evanescent field. A similar analysis may be applied to calculate the photonic response of periodic metal-dielectric multilayers, 
where the plasmon dispersion relations may be obtained from the Bloch theorem.

In the present case, an optical mode of a dielectric stack is the solution of the general eigenvalue equation:

$$
\nabla \times\left(\frac{1}{\varepsilon} \nabla \times H\right)=\omega^{2} \mu H
$$

where the dielectric function and the field are defined for each layer, and the satisfaction of the usual boundary conditions is given. The given harmonic mode of the dielectric system is the eigenfunction $H$ for a given eigenvalue, which is proportional to the frequency of the mode. For a dielectric function with the following general form:

$$
\varepsilon=\varepsilon(z)=\sum_{i} \varepsilon_{i+1} \Theta\left(z-z_{i}\right) \Theta\left(z_{i+1}-z\right),
$$

where $\Theta$ is the Heaviside function, if one can define, for a specific set of $\varepsilon_{i}$, a unit cell so that the same dielectric pattern can be repeated, one may then consider the following general (on-axis) solution or mode:

$$
H(x, y, z)=h(x, y, z) e^{j K z},
$$

which represents a Bloch state if $h$ is $z$-periodic, in analogy with the electron wave function in a solid in the presence of a periodic potential. Ideally, following the standard description of a 1D photonic crystal, we require

$$
\varepsilon(z+\Gamma)=\varepsilon(z),
$$

where $\Gamma$ is the period. Further interest is in the regime where $\Gamma \sim \lambda$, with $\lambda$ denoting the wavelength. Clearly, in the present case, $i$ is finite and we neither have a full translational symmetry, nor a partial one that scales with $\lambda$. For a medium with large $i$, we also note that, for off-axis modes, if the angle of incidence is larger than a critical angle, the Bloch mode will not propagate in the $z$ direction but in the perpendicular direction.

For a similar situation arising in mechanical systems described by an ordinary differential equation, the state is referred to as the Floquet mode [49]. Thus, the notion of the Bloch wave here is to emphasize the quasiperiodicity of the modulation of the dielectric function. Takayama et al. further reviews Bloch surface waves in close comparison with Tamm waves or optical Tamm states [48], which also require a (truncated) periodic permittivity.

For an alternating dielectric function, the resulting multilayer structure or the one-dimensional photonic crystal can form a Bragg mirror. Unlike an ideal one-dimensional photonic crystal, due to the finite length of the repeated layers, the periodicity in the dielectric function is not complete. Therefore, the field and their energy density may not exhibit a full symmetry in the $z$ direction. Although it is not the main focus of our current presentation, it may be possible to define a critical number of layers below which the periodicity may be sufficiently compromised for the results to differ significantly from the Bloch formulation. Nevertheless, even if the dielectric stack possesses a weak periodicity, a photonic band gap can still be formed, and its size can be estimated using perturbation theory. Notice however that at the top surface, the application of the ZAL breaks the symmetry and periodicity and a photonic mode can be localized there, forming a surface state.

To proceed, we note the following additional points, which necessitate use of another formalism. The first point is connected with the illumination beam coming from a superstrate, which breaks again the symmetry of the geometry and leads us to consider spatial frequencies that are not necessarily the modes of the structure. The second point pertains to the TIR regime that occurs in our lossless dielectric multilayers and implies that the reflection is unity in a whole spatial frequency band (above the refraction-angle limit). This is valid regardless of the design of the multilayer (the TIR limit is only connected with the refractive index of the output medium); therefore, the concept of band gap is irrelevant for this geometry. Eventually the response of finite nonperiodic multilayers can be classically modelized with the admittance formalism (see next section), whether the field is a mode of the structure or not, and whether the illumination comes from the extreme media or not.

Electromagnetic modes within 1D planar dielectric multilayers characterize fields, which satisfy the boundary conditions in the absence of sources. Their amplitude distribution in the harmonic regime can be written as

$$
E_{m}(x, z)=A_{m}(z) e^{j \sigma_{g, m^{x}}},
$$

with $\sigma_{g, m}$ a modal spatial pulsation. From the admittance formalism we note that $[50,51]$ the modal spatial pulsations $\sigma_{g, m}$ are the real poles of the reflection factor $r(\sigma)$ of the dielectric multilayer extended at higher frequencies (above the free-space limit), that is, $1 / r\left(\sigma_{g, m}\right)=0$, and that these poles (when they exist) lie in the frequency range given by

$$
\max \left(k_{0}, k_{s}\right)<\sigma_{g, m}<\max \left(k_{i}\right) \quad \text { with } \quad k_{i}=2 \pi n_{i} / \lambda,
$$

with $\lambda$ the wavelength, $n_{0}$ and $n_{s}$ the indices of superstrate and substrate respectively, and $n_{i}$ the stack indices. The field of a mode can be trigonometric or hyperbolic within the multilayer, but it is evanescent (with exponential decrease) in the extreme media (superstrate and substrate). Hence, these modes are confined within the stack and carry energy in the transverse directions (perpendicular to $z$ ), with no loss in the extreme media assumed to be nonabsorbing. Note for this modal geometry that the extended reflection function is not bounded since it is not involved in an energy balance.

If we now illuminate the multilayer under TIR, we lose the previous (source-free) mode configuration. The 
reflection poles cannot be real anymore since reflection is now bounded and involved in an energy balance in the form $R+A=1$, with $R$ and $A$ the energy coefficients of reflection and absorption. Hence, due to the modification of boundary conditions, the previous modal constraint $(1 / r=0)$ vanishes and there is no more discretization of frequencies. Actually, these frequencies can now be arbitrarily adjusted at low spatial frequencies with the illumination conditions. However, poles may still exist in the reflection coefficient, but they are necessarily complex (even with zero absorption). At specific frequencies and under the assumption of slight imaginary indices of the thin-film materials, these complex poles $\left(\sigma_{m}\right)$ may have slight imaginary values $\sigma^{\prime \prime}{ }_{m}$ together with real parts $\sigma^{\prime}{ }_{m}$ very close to the previous modal frequencies $\sigma_{g, m}$, that is

$$
\sigma_{m}=\sigma_{m}^{\prime}+j \sigma_{m}^{\prime \prime} \quad \text { with } \quad \sigma_{m}^{\prime} \approx \sigma_{g, m} \quad \text { and } \quad \sigma_{m}^{\prime \prime} / \sigma_{m}^{\prime} \ll 1 \text {. }
$$

These real frequencies, $\sigma_{m}^{\prime}$, are those where resonances occur and may create giant field enhancement in the TIR regime, and we recall in the next section how these enhancements can be chosen, designed, and optimized. The fields at the TIR resonances in our case may be viewed as different from the modal guided waves in photonic crystals since the total incident light is reflected (rather than coupled within the stack). Nevertheless, they exhibit distributions in the stack and substrate that resemble Bloch modes. We keep in mind that under TIR the field is evanescent in the substrate, while the incident and reflected beams interfere at the input surface of the multilayer, with $R=1$ for lossless dielectric materials. To summarize the above discussions, we assert that the description of the fields within our system bears both similarities and differences with a photonic crystal composed of a 1D periodic slab embedded between two homogenous media (the superstrate and the substrate in Fig. 1), a system that can be described using the Bloch-wave properties.

\section{B. Field enhancement for sensing applications}

Field enhancements usually occur in the spacer layers of multidielectric Fabry-Perot-type filters [52]. Such an enhancement increases as approximately $\beta^{2 N}$ with $\beta$ being the ratio of the refraction indices of the two materials that constitute the filter, and $2 \mathrm{~N}$ the number of thin-film layers [1]. This effect is classically related to the high reflectivity of the two mirrors on each side of the spacer layer, in addition to a phase-matching condition [41]. However, this field enhancement takes place within the bulk of the filter, which cannot be used for sensor applications, where the field maxima must be close to the output surface of the component. Hence, another design must be found for sensing applications (Fig. 1). One idea consists in keeping one multidielectric mirror (on the illumination side) and

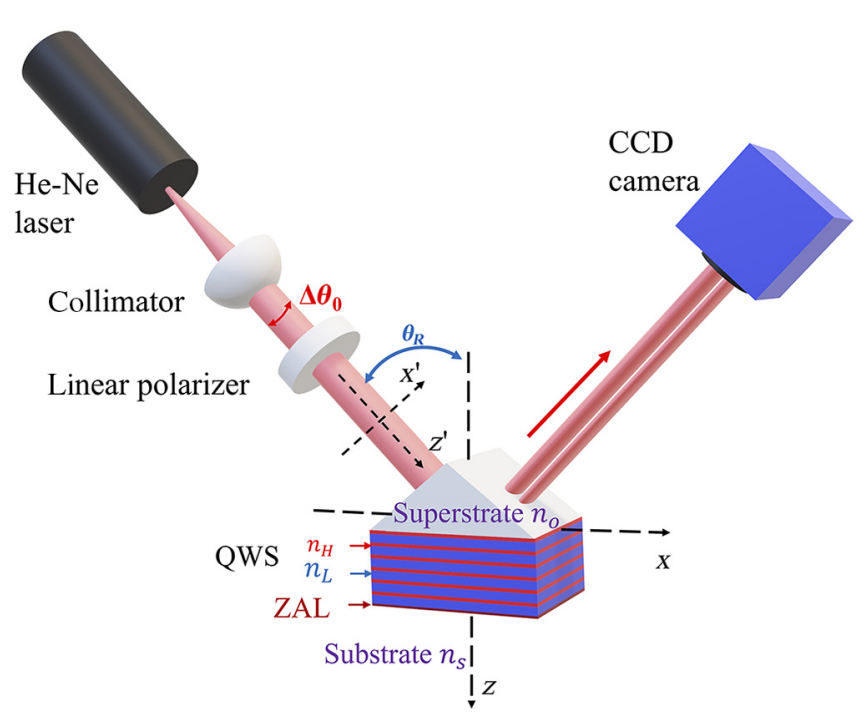

FIG. 1. Schematics of the experiment set up and a multidielectric stack on a prism, with a ZAL layer between the substrate and the quarter-wave mirror. An incoming beam making an angle $\theta_{R}$, resonantly couples into a multidielectric stack composed of a quarter-wave structure (QWS), ending with a zero-admittance layer. $x$ and $z$ are the coordinates of observer, and $x^{\prime}$ and $z^{\prime}$ are the coordinates of Gaussian beam.

taking advantage of the TIR regime to suppress the second mirror. This TIR procedure first requires working at high incidence angle (above the critical angle), so that the first mirror must be matched for this incidence (details are given in the next section). The second mirror is provided by the semi-infinite substrate since reflection is total at the output surface (reflection is unity under TIR). Then a thin layer is inserted between these two mirrors (the multidielectric mirror and the substrate), and a matching condition is adjusted, which relates the two mirrors and this spacer layer. With this procedure we are able to retrieve a field amplification similar to that of a Fabry-Perot filter, except that the enhancement occurs in the neighborhood on the substrate, and that the field is evanescent in the substrate (transmission is zero). We get into more detail in the next section.

\section{Calculation of the field enhancement}

We now recall the principles of ZAL [1] to design giant resonances in such multilayers. We consider dielectric materials that may be produced by high-energy deposition technologies [45-47]. Such materials are known to possess imaginary indices that are lower than $10^{-5}$. Therefore, absorption may be neglected, i.e., $A \approx 0$. As depicted in Fig. 1, we consider two quarter-wave multidielectric $Q_{m}$ structures in series:

$$
\begin{aligned}
& Q_{1}=S_{\mathrm{up}}(\mathrm{LH})^{N} P_{\mathrm{ZAL}} S_{\mathrm{ub}}, \\
& Q_{2}=S_{\mathrm{up}}(\mathrm{HL})^{N} P_{\mathrm{ZAL}} S_{\mathrm{ub}}
\end{aligned}
$$


where $H$ and $L$, respectively, denote high- and lowindex quarter-wave layers, and $(\mathrm{LH})^{N}$ means that the $\mathrm{LH}$ sequence is repeated $N$ times. Further, $P_{\mathrm{ZAL}}$ is an additional layer, $S_{\mathrm{ub}}$ denotes the emergent medium (substrate), while $S_{\text {up }}$ represents the incident medium (superstrate). The structures $Q_{m}$ are said to be quarter-wave dielectric mirrors since they are currently used to reflect light at a specific design wavelength $\lambda_{R}$. The $H$ and $L$ layers are therefore index matched for oblique illumination $\theta_{R}$ at the design wavelength $\lambda_{R}$, that is

$$
n_{i} e_{i} \cos \theta_{R}=\lambda_{R} / 4,
$$

with $n$ and $e$ being the indices and thicknesses of the films, and $\theta_{R}$ the illumination angle in the superstrate (incident medium), with $n_{i} \sin \theta_{i}=$ constant. To summarize, the $Q_{m}$ structures are classical quarter-wave mirrors matched for the illumination conditions $\left(\theta_{R}, \lambda_{R}\right)$. We assume that these illumination conditions satisfy the condition:

$$
k_{\text {sub }}<\sigma<k_{L}<k_{H},
$$

where the spatial pulsation (identical for all media) is given by $\sigma=k_{i} \sin \theta_{i}$ with $k_{i}=2 \pi n_{i} / \lambda$. Such a condition first allows angles $\left(\theta_{H}, \theta_{L}\right)$ to be defined in each film medium, so that the field is the sum of trigonometric functions (rather than hyperbolic) in these media. Furthermore, condition $k_{\text {sub }}<\sigma$ is required for TIR to occur, so that the field is evanescent (hyperbolic) in the substrate. Hence, no Poynting flux is carried through the substrate, so that reflection is unity for transparent stacks.

Following the admittance formalism [41], the matrix transfer of these multilayers follows at the design wavelength:

$$
\begin{aligned}
& Q_{1}=\left(M_{L} M_{H}\right)^{N}=(-1)^{N}\left(\begin{array}{cc}
\beta^{N} & 0 \\
0 & \beta^{-N}
\end{array}\right), \\
& Q_{2}=\left(M_{H} M_{L}\right)^{N}=(-1)^{N}\left(\begin{array}{cc}
\beta^{-N} & 0 \\
0 & \beta^{N}
\end{array}\right),
\end{aligned}
$$

where $N$ represents the number of doublets (HL or LH), $p=2 N$ the number of quarter-wave layers, and $\beta$ denotes the ratio of the effective indices for both TE and TM polarizations:

$$
\begin{aligned}
\beta & =\tilde{n}_{H} / \tilde{n}_{L} \\
\tilde{n}_{i} & =\frac{n_{i} k_{i}}{\alpha_{i}}=n_{i} / \cos \theta_{i} \quad \text { for TE polarization, } \\
\tilde{n}_{i} & =\frac{n_{i} \alpha_{i}}{\alpha_{i}}=n_{i} \cos \theta_{i} \quad \text { for TM polarization, } \\
\alpha_{i} & =\sqrt{k_{i}^{2}-\sigma^{2}}=k_{i} \cos \theta_{i},
\end{aligned}
$$

Eventually, the enhancement is given by

$$
\begin{aligned}
& \left|\frac{E_{s}}{E_{0}{ }^{+}}\right|^{2}=\left|\frac{2 \beta^{N} \tilde{n}_{0}}{Y_{s}+\tilde{n}_{0} \beta^{2 N}}\right|^{2} \text { for } Q_{1}=(\mathrm{LH})^{N} S, \\
& \left|\frac{E_{s}}{E_{0}{ }^{+}}\right|^{2}=\left|\frac{2 \beta^{N} \tilde{n}_{0}}{\tilde{n}_{0}+Y_{s} \beta^{2 N}}\right|^{2} \text { for } Q_{2}=(\mathrm{HL})^{N} S,
\end{aligned}
$$

where $S$ designates the substrate, $Y_{S}$ the substrate admittance, $E_{0}{ }^{+}$the tangential incident field, and $E_{s}$ the tangential field at the substrate surface (output surface). Following Eqs. (17) and (18), we observe that the enhancement tends to zero (irrespective of $\beta$ ) with the layer number $N$. However, this asymptotic behavior would be modified if the substrate admittance were zero; this is not possible at this step since $Y_{s}$ is given by the substrate effective index in Eqs. (14) and (15). Indeed, in the substrate the field is not stationary (but progressive evanescent) so that the admittance reduces to the effective index. For that reason, we consider the addition of a single layer $(p)$ on this substrate, the function of which is to cancel the admittance $\left(Y_{p-1}=0\right)$. While no solution can be found in the classical free-space regime $\left(\sigma<k_{\text {sub }}\right)$, we obtain in the TIR regime

$$
\tan \left(\alpha_{p} e_{p}\right)=\Im\left(Y_{s}\right) / \tilde{n}_{p} .
$$

Equation (19) provides the optical thickness $n_{p} e_{p}$ of the additional ZAL layer. Eventually, with this ZAL layer inserted between the substrate and the $Q_{i}$ structure, the enhancement now yields at the top surface of the ZAL layer:

$$
\begin{aligned}
&\left|\frac{E_{s}}{E_{0}{ }^{+}}\right|^{2}= \frac{4}{\beta^{2 N}} \text { for } Q_{1}=(\mathrm{LH})^{N} P_{\mathrm{ZAL}} S \text { and } \\
& \text { TM polarization, } \\
&\left|\frac{E_{S}}{E_{0}{ }^{+}}\right|^{2}=4 \beta^{2 N} \text { for } Q_{2}=(\mathrm{HL})^{N} P_{\mathrm{ZAL}} S \text { and }
\end{aligned}
$$

TE polarization.

We see that the ZAL thickness is very slight, so that the enhancement at the output substrate surface does not need to be corrected (to first order). Hence, according to the $\beta$ powers in Eqs. (19) and (20), huge overintensities may occur in the stack, and this can be designed for arbitrary illumination conditions (wavelength, polarization, and incidence). In what follows, these stacks are denoted ZAL stacks. Notice that the field is enhanced under the condition [1] that $\beta>1$ for TE polarization and $\beta<1$ for TM polarization. While the first (TE) condition is always satisfied in the angular range, the second (TM) 
condition requires working at large angles, that is, at angles greater than the Brewster angle [53]:

$$
\sigma>\sigma_{c}, \quad \text { with } \quad \sigma_{c}=\frac{k_{H} k_{L}}{\sqrt{k_{H}^{2}+k_{L}^{2}}} .
$$

At this step, we stress on the fact that [1] the enhancement a priori increases with the number of layers (no upper bound in the matrix formalism). Actually, the field limitation [1] is forced by intrinsic and extrinsic factors. Intrinsic factors are nonlinearities and damage thresholds, and (even slight) absorption and scattering. Extrinsic factors are related to the incident illumination band passes (in divergence and wavelength) [54], and the accuracy of the multilayer design $[1,34]$. Such limitations were previously analyzed [34-47] in detail since they require a compromise leading to a limited number of layers, and a realistic enhancement around four decades.

\section{Theory of dielectric multilayers and transmission lines}

Similar to the analogy between the description of a mechanical system using classical equation of motion versus using circuit theory, there is an illuminating analogy between the description of certain optical systems using classical optics versus using transmission-line theory [55]. Examples include dielectric thin-film multilayers, slabs, channel optical waveguides, directional couplers, anisotropic waveguides, etc. More specifically, for example, in Ref. [56], they present the optical equivalent of an antireflection quarter-wave transformer, optical tunneling, FTIR (frustrated TIR), and other quantities of importance in filter design. Generally, identification of the salient quantities from each formulation facilitates the correspondence. For transmission lines, the voltage $V$ and current $I$ along the line, and for optical systems, the electric $E$ and magnetic $H$ fields can facilitate the connections. Therefore, propagation of the optical field through the presented stack and the propagation of a voltage pulse (or radio waves) along a transmission line can be described analogously if the optical fields $(E, H)$ are identified with the electrical quantities $(V, I)$. A lossless dielectric thin film is analogues to a lossless transmission line such that the optical thickness of the film is equivalent to the electrical length of the line. Similarly, the refractive index of the thin film and the admittance of the segment of the line are on equal footing. The refractive index of the substrate of the stack may be identified with the load admittance of the line, etc. Following such an analogy, we note that condition (18) translates into a condition for a segment of a transmission line connected to a termination impedance to have an infinite input impedance [see Eq. 2.44 [57], and equate the denominator to zero]. Likewise, Eqs. (11) and (12) correspond to the $\mathrm{ABCD}$ matrices of two transmission-line segments, with quarter wavelength, resulting in a voltage transformer. From this analogy, one may infer that we here implement a series of voltage transformers to enhance the voltage $V$ (corresponding to $E$ ) at the expense of the current $I$ (corresponding to $H$ ) since the total power does not change. The use of the ZAL then terminates the line with an open circuit. Finally, we note that optical polarization and incidence can be simulated [58] from the impedance of the transmission line via $\mathrm{ZTM}=\eta \cos \theta$ and $\mathrm{ZTE}=\eta / \cos \theta$.

\section{DIVERGENT BEAM REFLECTED BY A ZAL STACK}

Due to the extreme sensitivity of the ZAL stacks around their design wavelength, even low beam divergences play a key role in the response of the multilayer. In Fig. 2, we show the enhancement (four decades) of a ZAL multilayer versus the spatial pulsation $\sigma$ for a design of (HL) ${ }^{6} P_{\mathrm{ZAL}} S$ and for TE polarization, with $\lambda_{R}=632.8 \mathrm{~nm}$ and $\theta_{R}=45^{\circ}$. Materials have real indices $n_{H}=2.25$ and $n_{L}=1.49$. The ZAL layer is a high-index layer with thickness $e_{p}=9.77 \mathrm{~nm}$. Note that in the absence of refractiveindex dispersion, both angular and wavelength behavior can be deduced from the curve in Fig. 2, since we have $\Delta \lambda=\lambda \Delta \sigma / \sigma$ and $\Delta \theta=\Delta \sigma / \alpha$, with $\Delta \theta, \Delta \lambda$, and $\Delta \sigma$ the angle, wavelength, and frequency widths of the curves. The enhancement is given for a plane-wave calculation and shows an intrinsic bandpass $\Delta \sigma=2.163 \times 10^{3} \mathrm{~m}^{-1}$, that is, $\Delta \lambda_{R}=0.3 \mathrm{~nm}$ and $\Delta \theta_{R}=0.16 \mathrm{mrad}$.

Here, we choose to work with a He-Ne laser beam with a spectral bandpass of around $\Delta \lambda=2 \mathrm{pm}$, which is much less than that of the stack $\left(\Delta \lambda_{R}=0.3 \mathrm{~nm}\right)$. Therefore, the beam can be considered monochromatic. On the other hand, the divergence $\Delta \theta$ of the He-Ne beam is of the same

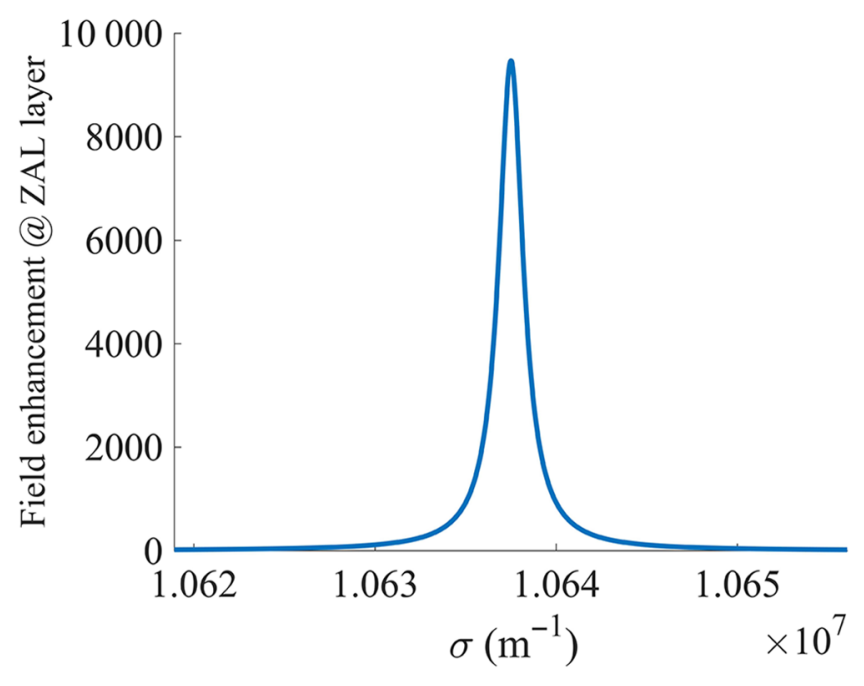

FIG. 2. Enhancement of an incoming TE polarized field versus spatial frequency. The stack is designed for a wavelength of $632.8 \mathrm{~nm}$ and an illumination angle of $45^{\circ}$. 
order of magnitude as that of the stack $\left(\Delta \theta_{R}=0.16 \mathrm{mrad}\right)$ so that its impact must be analyzed in detail. In this section, we use an exact spatial wave-packet formalism to analyze the wave front of the field reflected by the ZAL multilayer.

\section{A. Analytical calculation}

We consider a Gaussian beam illuminating the structure, as shown in Fig. 1. We work under the average conditions, $\theta_{0}=\theta_{R}$, and $\lambda_{0}=\lambda_{R}$, that is, the central incidence angle and wavelength are matched to the corresponding quantities at resonance. We express the incident wave as a two-dimensional (2D) monochromatic wave packet in the form

$$
E\left(x^{\prime}, z^{\prime}\right)=\int_{\sigma^{\prime}} A\left(\sigma^{\prime}\right) e^{j\left[\sigma^{\prime} x^{\prime}+\alpha\left(\sigma^{\prime}\right) z^{\prime}\right]} d \sigma^{\prime}
$$

with $A\left(\sigma^{\prime}\right)$ the amplitude distribution of the packet. Here, the $\left(x^{\prime}, z^{\prime}\right)$ coordinates are beam related $\left(z^{\prime}\right.$ is the average propagation direction, see Fig. 1) so that the amplitude function $A\left(\sigma^{\prime}\right)$ has a maximum modulus at $\sigma^{\prime}=0$. In other words, Eq. (23) is given for a wave packet written in its intrinsic coordinate system.

Assuming a Gaussian shape of the illuminated area in the form

$$
\left|E\left(x^{\prime}, z^{\prime}=0\right)^{2}\right|=\left|E_{0}\right|^{2} e^{-2\left(\frac{x^{\prime}}{L}\right)^{2}},
$$

with $2 L$ the dimension of the illuminated area, the amplitude function $A\left(\sigma^{\prime}\right)$ is related from the Fourier transform of Eq. (23) as

$$
\left|A\left(\sigma^{\prime}\right)\right|^{2}=\left|E_{0}\right|^{2}\left(\frac{L}{2 \pi}\right) e^{-\left(\frac{\sigma^{\prime} L}{2 \pi}\right)^{2}} .
$$

The quantity $L$ describing the Gaussian beam spot size at $1 / e$ is therefore linked to the beam divergence $\Delta \theta_{0}$ by

$$
\frac{\sigma_{\max }^{\prime} L}{2 \pi}=1 \stackrel{\text { yields }}{\longrightarrow} \Delta \theta_{0} \approx \lambda_{0} /\left(n_{0} L\right) .
$$

Extension of this wave-packet formulation to oblique incidence is usually performed with an integral similar to Eq. (23), in the form

$$
E\left(x^{\prime}, z^{\prime}\right)=\int_{\sigma^{\prime}} A\left(\sigma^{\prime}-\sigma^{\prime}{ }_{0}\right) e^{j\left[\sigma x^{\prime}+\alpha\left(\sigma^{\prime}\right) z^{\prime}\right]} d \sigma^{\prime}
$$

with $\sigma_{0}^{\prime}$ being the central frequency. However, Eq. (27) is not correct since it retains the shape of the illuminated area when incidence is modified. In order to correct Eq. (27), we modify the amplitude distribution by maintaining Eq. (23) but rotating $\theta_{0}$ of the $\left(x^{\prime}, z^{\prime}\right)$ plane to describe the same wave packet in the $(x, z)$ plane at an average oblique incidence $\theta_{0}$ on the sample. The change of coordinates in Eq. (23) leads to

$$
\begin{aligned}
E(x, z)= & \int_{\sigma} \frac{1}{\alpha}\left(\sigma \sin \theta_{0}+\alpha \cos \theta_{0}\right) A\left(\sigma \sin \theta_{0}-\alpha \cos \theta_{0}\right) \\
& \times e^{j[\sigma x+\alpha(\sigma) z]} d \sigma
\end{aligned}
$$

where $\sigma=\sigma^{\prime} \cos \theta_{0}+\alpha^{\prime} \sin \theta_{0}$ and $\alpha=\alpha^{\prime} \cos \theta_{0}-$ $\sigma^{\prime} \sin \theta_{0}$, so that $\sigma$ is in the range $\left[k_{0} \sin \left(\theta_{0}-\Delta \theta_{0}\right), k_{0}\right.$ $\left.\sin \left(\theta_{0}+\Delta \theta_{0}\right)\right]$. Thus, the elliptical shape of the illumination region with oblique incidence is now attained. Then, the reflected beam is described by a relation similar to Eq. (28) but one in which the new amplitude function is weighted at each frequency by the reflection coefficient $r(\sigma)$, that is

$$
\begin{aligned}
E_{r}(x, z)= & \int_{\sigma} \frac{1}{\alpha} r(\sigma)\left(\sigma \sin \theta_{0}-\alpha \cos \theta_{0}\right) A\left(\sigma \sin \theta_{0}\right. \\
& \left.+\alpha \cos \theta_{0}\right) \times e^{j[\sigma x-\alpha(\sigma) z]} d \sigma .
\end{aligned}
$$

In the absence of absorption, we note

$$
r(\sigma)=e^{j \phi(\sigma)}
$$

as the TIR condition yields $|r(\sigma)|=1$.

\section{B. Numerical calculation}

Equation (29) is implemented to calculate the beam wave front reflected by a ZAL stack. We design a $(\mathrm{HL})^{6} H_{\mathrm{ZAL}} S$ quarter-wave stack (identical to that of Fig. 2), with high index $n_{H}=2.25$ and low index $n_{L}=1.49$

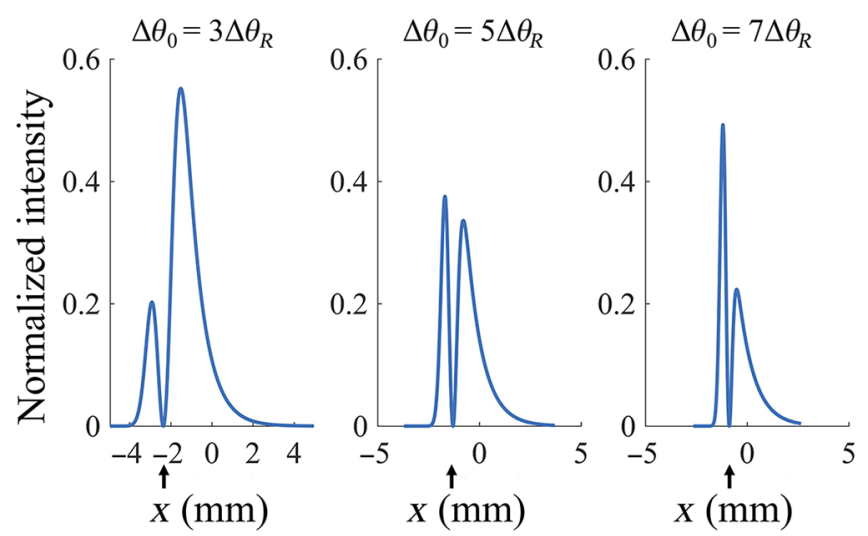

FIG. 3. Calculated spatial distribution of intensity in the reflected field profile registered in the CCD plane for various angular divergence $\Delta \theta_{0}$. The typically observed canceled field region, appearing in experiments as a dark line (black arrows) in the reflected beam spatial profile is clearly retrieved, as shown in the intensity plots. They are normalized to the maximum intensity of the incident beam. 

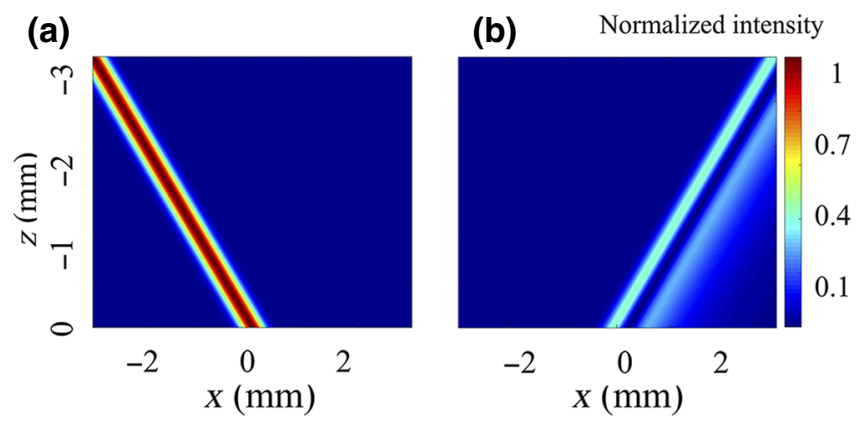

FIG. 4. Calculated distribution of the incident (a) and reflected (b) fields in the incidence plane at the resonance angle. Case of divergence $\Delta \theta_{0}=5 \Delta \theta_{R}=0.80 \mathrm{mrad}$.

at the illumination wavelength $\lambda_{R}=632.8 \mathrm{~nm}$ and angle $\theta_{R}=45^{\circ}$ for TE polarization. The incident medium is glass (BK7) with $n_{0}=1.52$ and the emergent medium is air $\left(n_{s}=1\right)$. There is a $9.77 \mathrm{~nm}$ ZAL layer with $n_{H}=2.25$ between the QWS and the substrate to cancel the admittance. High- and low-index thin-film materials are $\mathrm{Nb}_{2} \mathrm{O}_{5} / \mathrm{SiO}_{2}$, respectively.

The results are given in Fig. 3 and show how the spatial distribution of intensity depends on the angular beam divergence. The calculation is performed using spatial scales corresponding to a CCD matrix in a plane normal to the average reflection and positioned at $25 \mathrm{~cm}$ from the structure. We observe a clear modification of the reflected beam with divergence. In particular, we retrieve the wellknown [55] dark line region, which is characteristic of field cancellation in the detector area, due to destructive interferences (and not to absorption) or shift rays. Also, we notice the shift of the beam from one figure to another, as the result of a Goos-Hänchen [59] effect. These results show that the divergence of the incident beam should be properly suited to the resonance of the stack, since it determines the parts of transferred energy in the CCD area. Accordingly, a properly matched divergence would enhance the phenomena. (a)

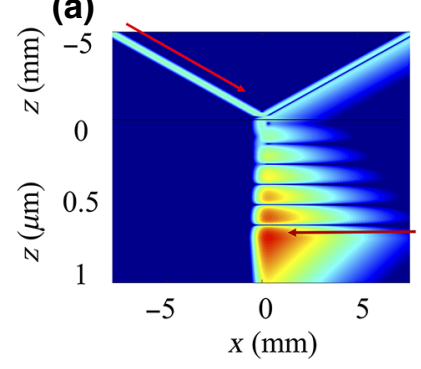

(b) Normalized intensity

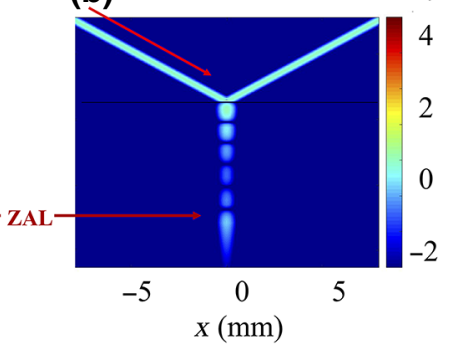

FIG. 5. Local field intensity visualized in logarithmic scale. The wave vector of the incident field and the location of the ZAL layer are annotated for clarity. (a) is obtained at the resonance angle with a $10^{3}$ enhancement, while (b) is out of resonance with a field enhancement around unity.
For a specific divergence $\Delta \theta_{0}=5 \Delta \theta_{R}=0.80 \mathrm{mrad}$, the field is visualized further in the whole incidence plane (along propagation) in Fig. 4, which now allows us to directly compare the incident and reflected beams at the resonance. We notice again the dark region of the reflected beam along propagation. Similarly, the depth distribution of the field in the stack can be examined, as shown in Fig. 5(a) at the resonance. These figures show both the enhancement in the bulk and the associated lateral asymmetry. For comparison, the enhancement is also plotted in Fig. 5(b) for an incidence $\left(46^{\circ}\right)$ different from the resonance angle $\left(45^{\circ}\right)$. Notice in all figures that the field is evanescent in the substrate.

\section{FABRICATION OF THE ZAL STACK}

As proof of concept, the designed structure is fabricated using plasma-assisted electron-beam deposition (Bühler SYRUSpro710) with residual initial pressure approximately $10^{-6}$ mbar. As an embodiment of the ZAL stack designed in the previous section, that is $\Delta \theta_{R}=0.16 \mathrm{mrad}$ and $\Delta \lambda_{R}=0.3 \mathrm{~nm}$, we prepare a stack consisting of six bilayers of $\mathrm{Nb}_{2} \mathrm{O}_{5}(79.31 \mathrm{~nm}) / \mathrm{SiO}_{2}(154.02 \mathrm{~nm})$, as highand low-index materials, respectively, and an 8.5-nm $\mathrm{Nb}_{2} \mathrm{O}_{5}$ layer as the zero-admittance layer. The ZAL thickness is different from that of Fig. 2 because we use the indices that are measured (see further in text), which show slight differences with the theoretical indices of Fig. 2. Pellets made of $\mathrm{Nb}_{2} \mathrm{O}_{5}$ and $\mathrm{SiO}_{2}$ are, respectively, placed into $\mathrm{Mo}$ and $\mathrm{Cu}$ crucibles. Deposition is achieved though e-beam evaporation of the raw materials at a rate of $0.25 \mathrm{~nm} / \mathrm{s}$ for $\mathrm{Nb}_{2} \mathrm{O}_{5}$ and $0.5 \mathrm{~nm} / \mathrm{s}$ for silica. The prisms to be coated are made of N-BK-7 with $n_{0}=1.5151$ at $632.8 \mathrm{~nm}$. All samples are placed on a rotating substrate holder at a distance of approximately $600 \mathrm{~mm}$ from the ebeam source in order to achieve high uniformity of $\pm 0.5 \%$ over a 100-mm diameter aperture. To guarantee dense layers with no porosity, an advanced oxygen plasma source is directed towards the samples during deposition, enabling the growth of dense and fully oxidized layers.

The refractive-index dispersion of each thin-film material is determined on previously fabricated single layers to accurately model and predict the behavior of the multilayer structures. The procedure consists in measuring transmittance and reflectance spectra of the samples with a spectrophotometer (Perkin Elmer Lambda 1050), before implementing reverse engineering to extract dispersion properties of the real and imaginary parts of the refractive index of each material [60]. A precision better than $10^{-3}$ could be achieved for the real part of the refractive index while negligible absorption $\left(k \ll 10^{-4}\right)$ is demonstrated for both materials in the visible and near-IR range. At the resonance measurement wavelength, i.e., $632.8 \mathrm{~nm}$, the real indices are $n_{L}=1.484$ and $n_{H}=2.264$. These measured index values are used for the ZAL design. 


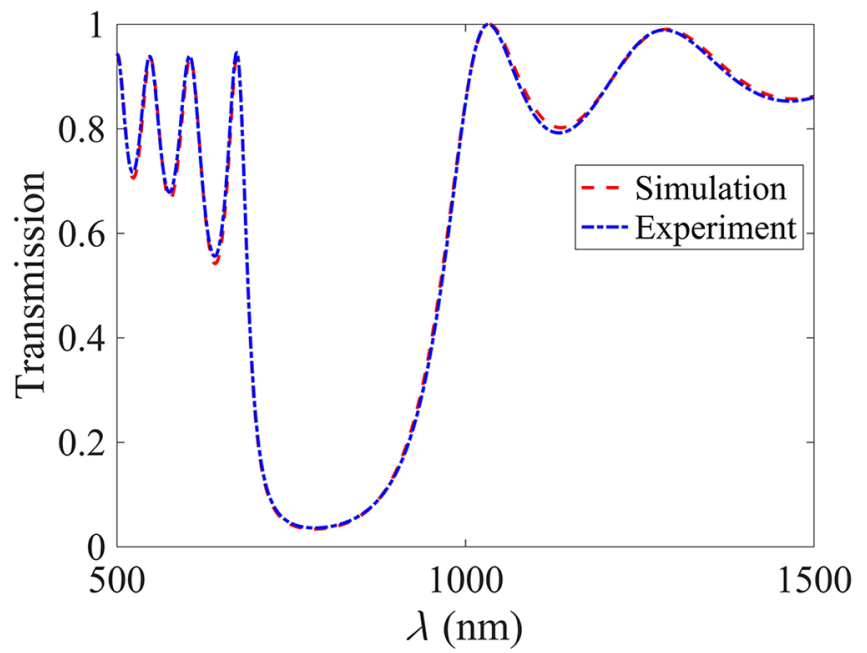

FIG. 6. Comparison of the experimentally measured and theoretically computed reflection spectra of the ZAL stack system. Excellent agreement is achieved. A normal illumination is considered on the witness sample (see text).

An in situ optical thickness monitor (Bühler OMS 5000 ) is used to minimize errors in the thickness of each deposited layer with respect to their designed values. This monitoring strategy can not be performed directly on the ZAL sample deposited on the prism, which works in the TIR regime (no spectrophotometric information is available under TIR to check the design since reflection is total for multidielectrics). Hence, we use a real-time single wavelength monitoring strategy [61] in transmittance mode on a witness glass substrate (without prism). The results are checked with the spectral measurements of reflectance recorded on the monitoring (witness) sample at quasinormal illumination. The resulting experimental data are plotted in Fig. 6 together with the calculation results for the ZAL design at $632.8 \mathrm{~nm}$ but for normal illumination. We observe that, over a broad spectral range of several hundreds of nanometers, the average error between theoretical and experimental profiles on the witness substrate is $<1 \%$, confirming that the fabricated stack is very close to the required design. Postdeposition analysis of the spectral transmittance shows that the equivalent average random errors on the thickness of each layer of the quarterwave stack is below $0.5 \%$. Concerning the ZAL thickness, which behaves in a different way, the accuracy is estimated to be approximately $5 \%$. It is noticed that errors in the ZAL thickness design can be compensated by adjusting the incidence angle on the prism.

\section{METROLOGY OF THE REFLECTED BEAM WAVE FRONT}

To characterize the reflected beam of the previous ZAL sample, we employ a TE polarized He-Ne laser beam with a wavelength of $632.8 \mathrm{~nm}$ and a linewidth of $\Delta \lambda=2 \mathrm{pm}$.
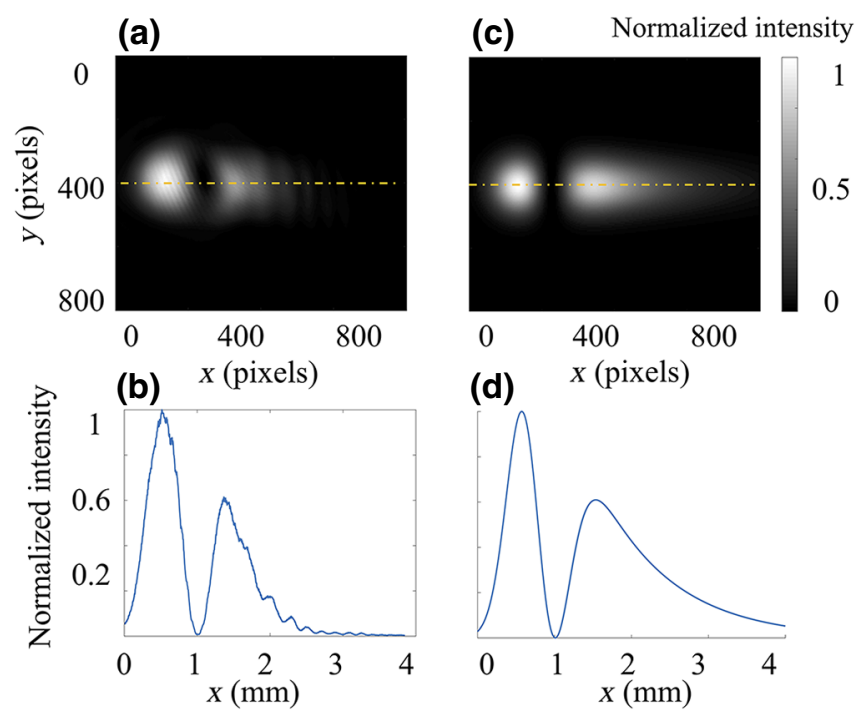

FIG. 7. Comparison of experimental (left) and theoretical (right) results for the spatial distribution of the reflected beam at the resonance. (a) and (c) display reflected beam shape. (b) and (d) are the corresponding cross sections indicated in (a) and (c). Each pixel is $25 \mu \mathrm{m}^{2}$.

This incident beam is coupled into a single-mode optical fiber. The fiber output is collimated, and the resulting divergence is $0.993 \mathrm{mrd}$ (i.e., $0.056^{\circ}$ ). The reflected beam at the resonance is recorded on a CCD detector (Fig. 1) at the distance of $25 \mathrm{~cm}$.

The measurement result is shown in Fig. 7(a), and compared to the theoretically computed reflected beam profile, shown in Fig. 7(b). As can be seen, an excellent agreement is achieved for the spatial distribution of the measured reflected beam. All effects are consistently observed, including beam-shape modification, average beam shift, and canceled field region ("dark line"). Such an agreement now allows us to use the criterion of the reflected beam shape as an analytical method for chemical and biological measurements.

We now aim to carry out analytical measurements to demonstrate how a sensing signal may be generated. Therefore, we seek the detection of trace amounts of an analyte interfacing the output surface of the proposed structure. For this purpose, we prepared a new ZAL coating designed to operate in distilled water (rather than air). The design is $(\mathrm{HL})^{3} L_{\mathrm{ZAL}} S$. This stack is optimized for a resonance at $\lambda_{R}=632.8 \mathrm{~nm}$ and $\theta_{R}=63^{\circ}$, for TE polarization. Notice that the incidence angle is higher than the previous ones, for which reason the number of layers is reduced. Hence, we have three bilayers of $\mathrm{Nb}_{2} \mathrm{O}_{5}$ $(90.21 \mathrm{~nm}) / \mathrm{SiO}_{2}(407.61 \mathrm{~nm})$, as high- and low-index materials, respectively. The zero-admittance layer (117.73$\mathrm{nm} \mathrm{SiO}_{2}$ ) is calculated for a substrate of distilled water (at room temperature the refractive index of distilled water is $n=1.3319$ [62]). 
This new substrate configuration is easily achieved by the deposition of 30 droplets of distilled water on the ZAL layer. Recall that the ZAL plane is a horizontal plane (see Fig. 1). The droplets spread in the form of a thin water layer on the ZAL layer, which covers the illumination area. Due to the fast decrease of the evanescent wave in the water layer (146-nm penetration depth), the thickness of this water layer is long enough for the water to be considered as a semi-infinite substrate.

In a second step, we introduce trace amounts of a NaClwater mixture into the water layer. For this purpose, one additional droplet of a known saline water mixture $(1 \%$ $\mathrm{NaCl}$ in water with an index of 1.3339 [63]) is deposited on the water layer. A few minutes' delay is necessary to reach equilibrium with a quasihomogeneous layer. Assuming a linear variation at low concentration [64], the variation of effective index is evaluated around $\Delta n=6 \times 10^{-5}$.

The measurement results are shown in Fig. 8. With the introduction of the $\mathrm{NaCl}$ mixture, as can be seen, the beam profile undergoes significant changes when compared to that with only water. This preliminary experiment demonstrates the utility of the technique to detect index modifications of approximately $10^{-5}$. Further addition (one more droplet) of trace amounts of saline water results in an index modification of $\Delta n \approx 1.2 \times 10^{-4}$, and the results are also plotted in Fig. 8.

However, we observe in Fig. 8 that the baseline (curve A in red) does not match as well as in Fig. 7, since its minimum level is far from zero. This may originate from the roughness scattering of the multilayer, which is intense

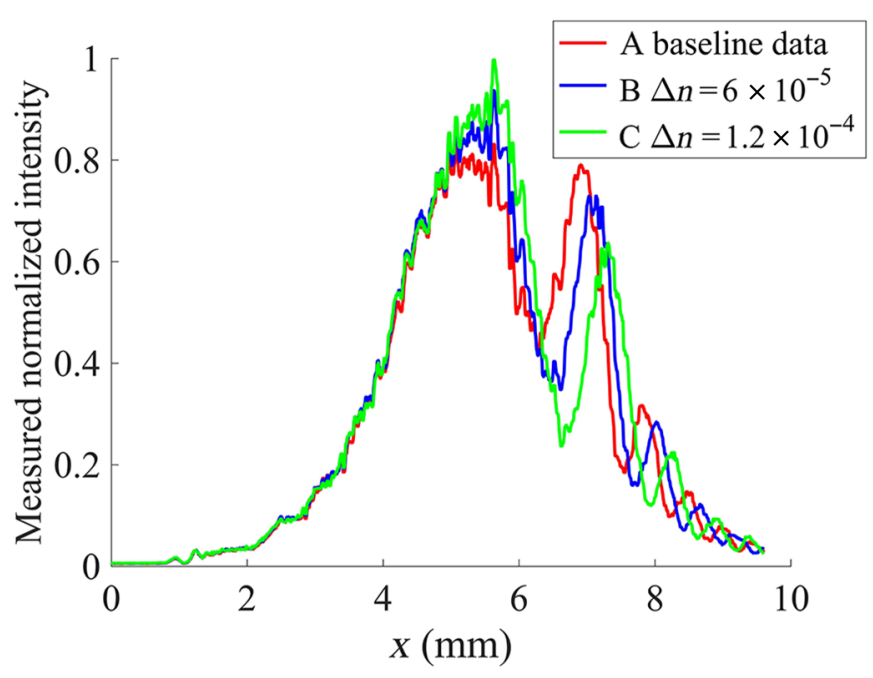

FIG. 8. Experimental measurement of the output beam profiles. The baseline data (A) represents the response to water, that is, before the introduction of the analyte. The response after the introduction of droplets of $1 \% \mathrm{NaCl}$-water mixture is labeled $\mathrm{B}$ and $\mathrm{C}$. An analysis of the data yields a change in the refractive index of $6 \times 10^{-5}$ and $1.2 \times 10^{-4}$ corresponding to an increase in the analyte concentration. at the resonance, since it is proportional to the stationary field at interfaces [40]. Hence, the scattering may alter the dark region and reduce the contrast of the baseline curve. Results may vary from one baseline curve to another, depending on the substrate and coating quality (roughness and cleanliness, local defects) that are different in Figs. 7 and 8. Furthermore, the enhancement in Fig. 7 is lower (around $10^{3}$ ) than that of Fig. 8 (around $10^{4}$ ), which may explain why the contrast is better in Fig. 7, due to a lower scattering.

To further support the experimental observations, in Fig. 9 we display the corresponding theoretical results and they show good qualitative agreement. Note at this step that additional differences may also originate from the accuracy of the design (recall that the ZAL thickness accuracy is $5 \%$ ). We observe that the calculated contrast variation is in opposition with the experimental one, which may again be attributed to the scattering effects. Indeed, scattering decreases with departure from the resonance (reduction of enhancement), so that a better experimental contrast is obtained far from the resonance (that is, with increasing values of $\Delta n$ ). This parasitic light at the resonance holds whatever the origins of scattering (surface roughness or bulk inhomogeneities), though surface scattering is known to be predominant in high-quality optical coatings deposited on polished substrates [50,51,65-69]. Relying on first-order electromagnetic theories [50,51,6569], the scattering surface currents are proportional to the stationary field at the stack interfaces, for which reason roughness-induced scattering is much higher at the resonance angle (by several orders of magnitude).

Another difference between Fig. 8 (experiment) and Fig. 9 (calculation) concerns the order of magnitude of the

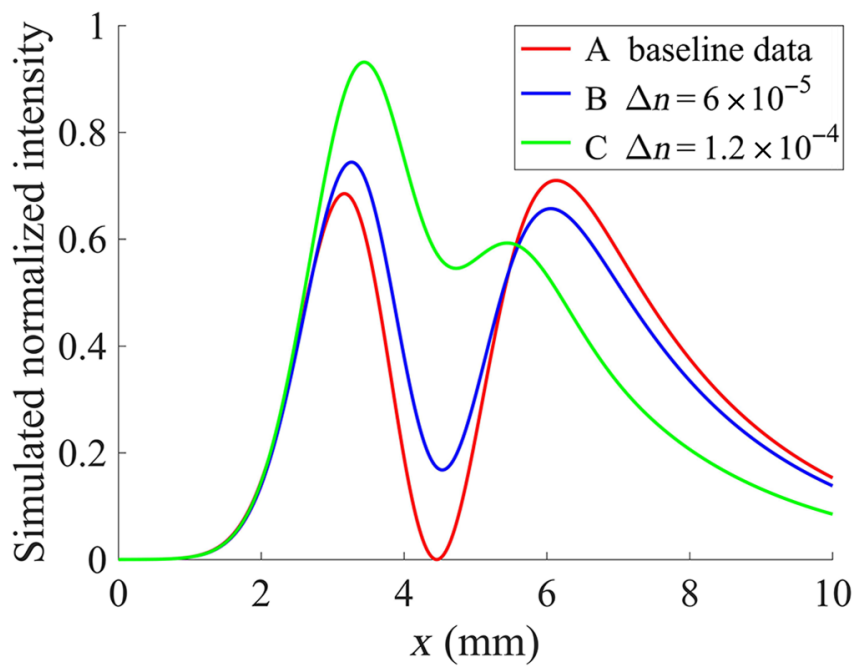

FIG. 9. Theoretical determination of the output beam profiles. A shift in the response is clearly discernible with the introduction of index changes employed in the experiments. 
lateral shifts ( $x$ coordinate). The calculated shift (between baseline and lower contamination curve) is around $100 \mu \mathrm{m}$, while the measured value is around $300 \mu \mathrm{m}$. Further work will be necessary to explain this difference. At this step one can incriminate a lack of accuracy in the divergence values or an additional deviation due to refraction at the prism output (not taken into account by the calculation) and/or slight errors in the multilayer design. Despite the noted variations, the results clearly demonstrate the utility of the ZAL technique for detection of trace amounts of elements. In the next section, we propose to quantify a detection limit.

\section{SENSITIVITY OF THE SENSING MECHANISM}

As mentioned before, the role of ZAL is to cancel the admittance, resulting in a huge enhancement with high sensitivity to the surrounding conditions. Then, once the refractive index of the substrate (formerly air) is slightly modified by trace amounts of impurities to be detected, the zero-admittance condition would not be valid anymore, resulting in a modification of the reflected wave front, which eventually constitutes the detection criterion. Here, we analyze and quantify the sensitivity of this sensing mechanism. Unlike other detection criteria, such as those of SPR, in which an angular or spectral shift of the absorption peak furnishes a sensing channel, here, the situation is different since absorption is zero. For that reason, we use the spatial profile of the reflected beam as a detection criterion. Thus, our sensing criterion is based upon the detection of a modification in the beam profile, which requires a pixelized receiver, as opposed to the measurement of a global absorption or reflection (which can be measured with a monobloc receiver).

Rather than considering a particular data on the beam profile (amplitude or position of the extrema, contrast of the dark region, edge fronts, etc.), we start with the most basic criterion, which is the normalized distance between the whole experimental and theoretical profiles. In order to simulate this distance, we denote by $R_{o}(x)$ and $R_{a}(x)$ the theoretically calculated reflection on the CCD area, where $R_{o}(x)$ is the original curve without trace impurities, and $R_{a}(x)$ is the calculated curve after addition of trace impurities (when the substrate has changed). The normalized distance $D$ between these two curves is written as

$$
D=\frac{1}{N} \sum_{n}\left[\frac{R_{a}\left(X_{n}\right)-R_{0}\left(X_{n}\right)}{R_{o}\left(X_{n}\right)}\right]^{2} .
$$

Furthermore, taking into account the accuracy of measurements, we consider that an easy-measurable departure from the $R_{0}$ reference (baseline curve without trace impurities) is given by $D>D_{\text {th }}=4 \%$, with $D_{\text {th }}$ the detection threshold. Notice here that we choose a very severe (high) threshold in regard to metrology values that can be used

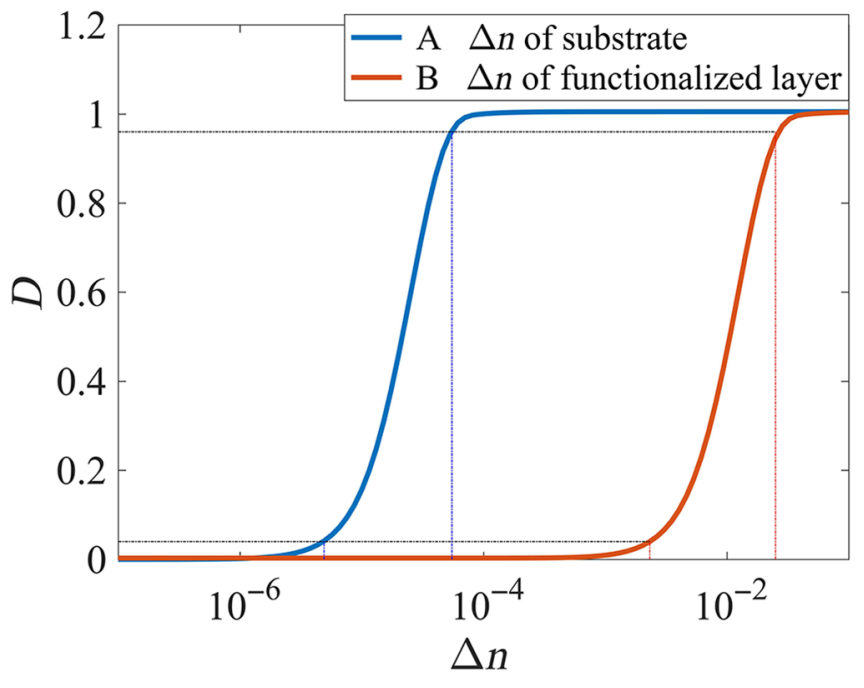

FIG. 10. Detection criterion D, as given by Eq. (31), as a function of the variation in the dielectric function $\Delta n$ of the substrate. We choose a sensing criterion of $4 \%$ difference with respect to the ZAL coating before contamination. Curve A corresponds to trace impurities in the (bulk) air substrate, while curve B corresponds to trace impurities in a 1-nm functionalized layer that is considered to be part of the ZAL coating.

(lower than 1\%). The results are given in Fig. 10. Curve A corresponds to the case when the trace impurity occurs in the whole substrate; that is for example, when the air substrate is replaced by a gas of real refractive index $n_{g}$. In Fig. 10 the distance $D$ is plotted versus the index difference $\Delta n=n_{g}-n_{\text {air }}$ and emphasizes a sensitivity of $\Delta n=5 \times 10^{-6}$. For completeness, we also plot curve $\mathrm{B}$, which represents the most common situation in sensing when the last ZAL layer is functionalized. A typical functionalization layer has thickness and refractive-index values close to $e_{f}=1 \mathrm{~nm}$ and $n_{f}=1.3$, respectively. Hence, in the calculation of curve $\mathrm{B}$, the thickness of the ZAL layer is redesigned to include this 1-nm functionalized layer via Eq. (19) where $\tilde{n}_{s}$ is replaced by $Y_{s}$. Therefore, the resulting stack includes the quarter-wave mirror, the ZAL layer and the functionalized layer, and this ensures that the stack properties are not modified. Upon trace-element incorporation, the refractive index of the functionalized layer is modified by a value $\Delta n$. The results are plotted in Fig. 10 (curve B) and reveal a $\Delta n$ sensitivity of $2.3 \times 10^{-3}$. Such a value obviously depends on the thickness of the functionalized layer and penetration range of the trace elements; here, we only plot one curve (corresponding to a $1 \mathrm{~nm}$ thickness of the functionalized layer) due to the fact that the trace elements are expected to roughly penetrate $1 \mathrm{~nm}$ in this layer. Now to conclude this section, we must stress on the fact that depending on the trace-element incorporation process, either the functionalized layer, the substrate, or both (substrate and functionalized layer) can incorporate the trace elements and therefore both curves A 
and B are necessary. Also, we keep in mind that the theoretical sensitivity obtained can be improved by changing the detection criteria (using a lower threshold for instance, or an extrema or edge-front criteria in the beam profile) or the detection channel (using phase information or light scattering).

\section{CONCLUSION}

Our parametric studies of the ZAL based upon the characteristics of the reflected beam profile enable evaluation of the sensitivity of planar ZAL multilayers. In addition to furnishing a detection channel, these studies can enable further optimization of the performance of the stack. For example, similar studies can assess the ability to further optimize the sensing response based on the use of beam profiles other than Gaussian. Employing the shape modification of the reflected beam at resonance as a detection criterion is motivated here by the ZAL stacks having negligible absorption. The exact calculation of the output beam shape changes upon reflection of the incoming beam on the ZAL coatings, including m-line [55] and Goos-Hänchen [59] effects, constitutes a robust method for evaluating other potential input beam shapes, albeit with an added complexity. This is evident from the successful comparison of theoretical and experimental data that validate the calculations presented. Assuming a sensitivity threshold of $4 \%$ (normalized value) on the changes of the reflected beam profile results in a refractive-index sensitivity of $\Delta n=5 \times 10^{-6}$ in the case of trace impurities addition to the (complete) bulk substrate, and of $\Delta n=2.3 \times 10^{-3}$ in the case of trace impurities addition to the 1-nm functionalization layer. These results demonstrate the potential of the proposed system for quantitative sensing. We will also investigate different criterion that could lead to even better sensitivity. We conclude that the ZAL coatings (planar multilayers without nanostructure) can be used with high sensitivity in the field of sensors. Future work will include integration with microfluidic systems [70] in order to develop and demonstrate a ZAL-based sensor system with different functional layers.

\section{ACKNOWLEDGMENTS}

The authors acknowledge the PSA group for their support through the OpenLab PSA/AMU (Automotive Motion Lab through the StelLab network), the ANRT for their support through the CIFRE program and the RCMO Group of the Institut Fresnel for the realization of the coatings. A.P. acknowledges support from the laboratory directed research and development (LDRD) program at the Oak Ridge National Laboratory (ORNL). This work is also partly supported by a grant from the PROCOREFrance/Hong Kong Joint Research Scheme sponsored by the Research Grants Council of Hong Kong and the
Consulate General of France in Hong Kong (Reference No. F-CityU108/16).

[1] C. Amra, M. Zerrad, F. Lemarchand, A. Lereu, A. Passian, J. Antonio Zapien, and M. Lequime, Energy density engineering via zero admittance domains in all-dielectric stratified materials, Phys. Rev. A 97, 023819 (2018).

[2] P. Yeh, A. Yariv, and A. Y. Cho, Optical surface waves in periodic layered media, Appl. Phys. Lett. 32, 104 (1978).

[3] W. Ng, P. Yeh, P. C. Chen, and A. Yariv, Optical surface waves in periodic layered medium grown by liquid phase epitaxy, Appl. Phys. Lett. 32, 370 (1978).

[4] P. Yeh, A. Yariv, and C.-S. Hong, Electromagnetic propagation in periodic stratified media. I. General theory, J. Opt. Soc. Am. 67, 423 (1977).

[5] A. Yariv and P. Yeh, Electromagnetic propagation in periodic stratified media. II. Birefringence, phase matching, and x-ray lasers, J. Opt. Soc. Am. 67, 438 (1977).

[6] N. Ashby and S. C. Miller, Interference theory of reflection from multilayered media, J. Opt. Soc. Am. 67, 448 (1977).

[7] E. R. Mendieta and P. Halevi, Electromagnetic surface modes of a dielectric superlattice: The supercell method, J. Opt. Soc. Am. B 14, 370 (1997).

[8] W. M. Robertson and M. S. May, Surface electromagnetic wave excitation on one-dimensional photonic band-gap arrays, Appl. Phys. Lett. 74, 1800 (1999).

[9] K. Mehrany, S. Khorasani, and B. Rashidian, Novel optical devices based on surface wave excitation at conducting interfaces, Semicond. Sci. Technol. 18, 582 (2003).

[10] J. Martorell, D. W. L. Sprung, and G. V. Morozov, Surface TE waves on 1D photonic crystals, J. Opt. A: Pure Appl. Opt. 8, 630 (2006).

[11] F. Michelotti, A. Sinibaldi, P. Munzert, N. Danz, and E. Descrovi, Probing losses of dielectric multilayers by means of Bloch surface waves, Opt. Lett. 38, 616 (2013).

[12] Jiřr' 'Homola, Sinclair S. Yee, and Günter Gauglitz, Surface plasmon resonance sensors: Review, Sens. Actuators, B: Chem. 54, 3 (1999).

[13] S. Yin and P. Ruffin, in Wiley Encyclopedia of Biomedical Engineering, edited by M. Akay, 2006).

[14] P. Munzert, N. Danz, A. Sinibaldi, and F. Michelotti, Multilayer coatings for Bloch surface wave optical biosensors, Surf. Coat. Technol. 314, 79 (2017).

[15] A. Shalabney and I. Abdulhalim, Electromagnetic fields distribution in multilayer thin film structures and the origin of sensitivity enhancement in surface plasmon resonance sensors, Sens. Actuators, A 159, 24 (2010).

[16] A. Sinibaldi, N. Danz, E. Descrovic, P. Munzert, U. Schulz, F. Sonntag, L. Dominici, and F. Michelotti, Direct comparison of the performance of Bloch surface wave and surface plasmon polariton sensors, Sens. Actuators, B 174, 292 (2012).

[17] Wan YuHang, Zheng Zheng, Shi XiaoGang, Bian YuSheng, and Liu JianSheng, Hybrid plasmon waveguide leveraging Bloch surface polaritons for sub-wavelength confinement, Sci. China Tech. Sci. 56, 567 (2013).

[18] Z. Sekkat, S. Hayashi, D. V. Nesterenko, A. Rahmouni, S. Refki, H. Ishitobi, Y. Inouye, and S. Kawata, Plasmonic 
coupled modes in metal-dielectric multilayer structures: Fano resonance and giant field enhancement, Opt. Express 24, 20080 (2016).

[19] R. H. Ritchie, Plasma losses by fast electrons in thin films, Phys. Rev. 106, 874 (1957).

[20] A. Passian, A. Wig, A. L. Lereu, P. G. Evans, F. Meriaudeau, T. Thundat, and T. L. Ferrell, Probing large area surface plasmon interference in thin metal films using photon scanning tunneling microscopy, Ultramicroscopy $\mathbf{1 0 0}$, 429 (2004).

[21] A. Passian, A. L. Lereu, A. Wig, F. Meriaudeau, T. Thundat, and T. L. Ferrell, Imaging standing surface plasmons by photon tunneling, Phys. Rev. B 71, 165418 (2005).

[22] E. Ozbay, Plasmonics: Merging photonics and electronics at nanoscale dimensions, Science 311, 189 (2006).

[23] A. Archambault, T. V. Teperik, F. Marquier, and J. J. Greffet, Surface plasmon Fourier optics, Phys. Rev. B 79, 195414 (2009).

[24] E. Kretschmann, The determination of the optical constants of metals by excitation of surface plasmons, Z. Phys. 241, 313 (1971).

[25] W. A. Challener, J. D. Edwards, R. W. McGowan, J. Skorjanec, and Z. Yang, A multilayer grating-based evanescent wave sensing technique, Sens. Actuators, B 71, 42 (2000).

[26] K. Toma, E. Descrovi, M. Toma, M. Ballarini, P. Mandracci, F. Giorgis, A. Mateescu, U. Jonas, W. Knoll, and J. Dostalek, Bloch surface wave-enhanced fluorescence biosensor, Biosens. Bioelectron. 43, 108 (2013).

[27] F. Frascella, S. Ricciardi, P. Rivolo, V. Moi, F. Giorgis, E. Descrovi, F. Michelotti, P. Munzert, N. Danz, L. Napione, M. Alvaro, and F. Bussolino, A fluorescent One-dimensional photonic crystal for label-free biosensing based on Bloch surface waves, Sensors 13, 2011 (2013).

[28] A. Sinibaldi, R. Rizzo, G. Figliozzzi, E. Descrovic, N. Danz, P. Munzert, A. Anopchenko, and F. Michelotti, A full ellipsometric approach to optical sensing with Bloch surface waves on photonic crystals, Opt. Express 21, 23331 (2013).

[29] A. L. Lereu, M. Zerrad, A. Passian, and C. Amra, Surface plasmons and Bloch surface waves: Towards optimized ultra-sensitive optical sensors, Appl. Phys. Lett. 111, 011107 (2017).

[30] F. Michelotti, R. Rizzo, A. Sinibaldi, P. Munzert, C. Wachter, and N. Danz, Design rules for combined labelfree and fluorescence Bloch surface wave biosensors, Opt. Lett. 42, 2798 (2017).

[31] A. Occhicone, A. Sinibaldi, F. Sonntag, P. Munzert, N. Danz, and F. Michelotti, A novel technique based on Bloch surface waves sustained by one-dimensional photonic crystals to probe mass transport in a microfluidic channel, Sens. Actuators, B 247, 532 (2017).

[32] Amin Ranjbar and Anthony Grbic, Broadband, Multiband, and Multifunctional All-Dielectric Metasurfaces, Phys. Rev. Appl. 11, 054066 (2019).

[33] Ruxue Wang, Junxue Chen, Yifeng Xiang, Yan Kuai, Pei Wang, Hai Ming, Joseph R. Lakowicz, and Douguo Zhang, Two-Dimensional Photonic Devices Based on Bloch Surface Waves With One-Dimensional Grooves, Phys. Rev. Appl. 10, 024032 (2018).

[34] A. L. Lereu, F. Lemarchand, M. Zerrad, D. Niu, V. Aubry, A. Passian, and C. Amra, Sensitivity of resonance properties of all-dielectric multilayers driven by statistical fluctuations, Opt. Express 29, 30654 (2019).

[35] L. Gao, F. Lemarchand, and M. Lequime, Exploitation of multiple incidences spectrometric measurements for thin film reverse engineering, Opt. Express 20, 15734 (2012).

[36] R. C. Nesnidal and T. G. Walker, Multilayer dielectric structure for enhancement of evanescent waves, Appl. Opt. 35, 2226 (1996).

[37] R. Sainidou, J. Renger, T. V. Teperik, M.-U. Gonzalez, R. Quidant, F. J, and Garcia de Abajo, Extraordinary Alldielectric light enhancement over large volumes, Nano Lett. 10, 4450 (2010).

[38] C. Ndiaye, F. Lemarchand, M. Zerrad, D. Ausserré, and C. Amra, Optimal design for $100 \%$ absorption and maximum field enhancement in thin-film multilayers at resonances under total reflection, Appl. Opt. 50, C382 (2011).

[39] C. Amra, C. Ndiaye, M. Zerrad, and F. Lemarchand, Optimal design for field enhancement in optical coatings, Proc. SPIE 8168, 8168 (2011).

[40] A. L. Lereu, M. Zerrad, C. Ndiaye, F. Lemarchand, and C. Amra, Scattering losses in multidielectric structures designed for giant optical field enhancement, Appl. Opt. 53, A412 (2014).

[41] H. Angus MacLeod, Thin Film Optical Filters (CRC Press, Florida, 2010).

[42] Alfred Thelen, Design of Optical Interference Coating (McGraw-Hill, New York, 1989).

[43] S. A. Furman and A. V. Tikhonravov, Basics of Optics of Multilayer Systems (World Scientific Publishing Co Pte Ltd, Singapore, 1996).

[44] Philip W. Baumeister, Optical Coating Technology (SPIE Publications, Washington, 2004).

[45] J. Ho, C. Chen, C. Huang, W. Lee, W. Liou, and C. Chang, Ion-assisted sputtering deposition of antireflection film coating for flexible liquid-crystal display applications, Appl. Opt. 44, 6176 (2005).

[46] M. Bischoff, T. Nowitzki, O. Voß, S. Wilbrandt, and O. Stenzel, Postdeposition treatment of IBS coatings for UV applications with optimized thin-film stress properties, Appl. Opt. 53, A212 (2014).

[47] D. Rademacher, B. Fritz, and M. Vergöhl, Origin of particles during reactive sputtering of oxides using planar and cylindrical magnetrons, Appl. Opt. 51, 927 (2012).

[48] O. Takayama, A. A. Bogdanov, and A. V. Lavrinenko, Photonic surface waves on metamaterial interfaces, J. Phys.: Condens. Matter 29, 463001 (2017).

[49] Pablo Gómez García and José-Paulino Fernández-Álvarez, Floquet-Bloch theory and its application to the dispersion curves of nonperiodic layered systems, Math. Probl. Eng. (2015).

[50] Claude Amra and Sophie Maure, Electromagnetic power provided by sources within multilayer optics: Free-space and modal patterns, J. Opt. Soc. Am. A 14, 3102 (1997).

[51] Claude Amra and Sophie Maure, Mutual coherence and conical pattern of sources optimally excited within multilayer optics, J. Opt. Soc. Am. A 14, 3114 (1997).

[52] M. Lequime, B. Gralak, S. Guenneau, M. Zerrad, and C. Amra, Negative-index materials: A key to "white" multilayer Fabry-Perot, Opt. Lett. 39, 1729 (2014).

[53] Brewster David IX. On the laws which regulate the polarisation of light by reflexion from transparent bodies. By 
David Brewster, LL. D. F. R. S. Edin. and F. S. A. Edin. In a letter addressed to Right Hon. Sir Joseph Banks, Bart. K. B. P. R. S105Phil. Trans. R. Soc.

[54] M. Zerrad, A. Lereu, C. N'diaye, F. Lemarchand, and C. Amra, Bandwidths limitations of giant optical field enhancements in dielectric multi-layers, Opt. Express 25, 14883 (2017).

[55] S. Monneret, et al., m-lines technique: Prism coupling measurement and discussion of accuracy for homogeneous waveguides, J. Opt. A: Pure Appl. Opt. 2, 188 (2000).

[56] R. Pieper, M. Shirvaikar, and J. Salvatierra, in 38th Southeastern Symposium on System Theory (2006).

[57] David Pozer, Microwave Engineering (Wiley, New Jersey, 2011).

[58] Sophocles J. Orfanidis, Electromagnetic Waves and Antennas (Prentice-Hall, New Jersey, 2003).

[59] F. Goos and H. Hänchen, Ein neuer und fundamentaler versuch zur totalreflexion, Ann. Phys. 436, 333 (1947).

[60] L. Gao, F. Lemarchand, and M. Lequime, Comparison of different dispersion models for single layer optical thin film index determination, Thin Solid Films 520, 501 (2011). Elsevier.

[61] Alexander V. Tikhonravov, Michael K. Trubetskov, and Tatiana V. Amotchkina, Optical Thin Films and Coatings (Second Edition) (Woodhead Publishing, Cambridge, 2018).

[62] Masahiko Daimon and Akira Masumura, Measurement of the refractive index of distilled water from the near-infrared region to the ultraviolet region, Appl. Opt. 46, 3811 (2007).

[63] Xingcan Li, Linhua Liu, Junming Zhao, and Jianyu Tan, Optical properties of sodium chloride solution within the spectral range from 300 to $2500 \mathrm{~nm}$ at room temperature, Appl. Spectrosc. 69, 635 (2015).

[64] N. An, B. Zhuang, M. Li, Y. Lu, and Z. Wang, Combined theoretical and experimental study of refractive indices of water-acetonitrile-salt systems, J. Phys. Chem. B 119, 10701 (2015).

[65] C. Amra, Light scattering from multilayer optics. I. Tools of investigation, J. Opt. Soc. Am. A 11, 197 (1994).

[66] C. Amra, Light scattering from multilayer optics. II. Application to experiment, J. Opt. Soc. Am. A 11, 211 (1994).

[67] C. Amra, C. Grèzes-Besset, and L. Bruel, Comparison of surface and bulk scattering in optical multilayers, Appl. Opt. 32, 5492 (1993).

[68] M. Zerrad, S. Liukaityte, M. Lequime, and C. Amra, Light scattered by optical coatings: Numerical predictions and comparison to experiment for a global analysis, applied optics, Opt. Soc. Am. 55, 9680 (2016).

[69] Marin Fouchier, Myriam Zerrad, Michel Lequime, and Claude Amra, Wide-range wavelength and angle resolved light scattering measurement apparatus, Opt. Lett. 45, 2506 (2020).

[70] Shelley D. Minteer, Microfluidic Techniques Reviews and Protocols (Humana Press, New Jersey, 2006). 